\title{
Large-scale sequencing combined with bioinformatics analysis reveals KLF8 an apoptosis repressor in hepatocellular carcinoma
}

Hao Xing

Chinese Naval Academy

Jin Zhang

Chinese Naval Academy

Kai-Lian Zheng

Chinese Naval Academy

Ming-Da Wang

Chinese Naval Academy

Jun Han

Chinese Naval Academy

Chao Li

Chinese Naval Academy

Zhen-Li Li

Chinese Naval Academy

Han Wu

Chinese Naval Academy

Lei Liang

Chinese Naval Academy

Meng-Chao Wu

Chinese Naval Academy

Feng Shen

Chinese Naval Academy

Tian Yang ( $\square$ yiqi9456312318@163.com )

Shanghai Jiao Tong University School of Medicine https://orcid.org/0000-0002-7227-2342

Research article

Keywords: KLF8, HCC, apoptosis, H3K27 acetylation

Posted Date: August 21st, 2019

DOI: https://doi.org/10.21203/rs.2.13333/v1 
License: (c) (i) This work is licensed under a Creative Commons Attribution 4.0 International License. Read Full License 


\section{Abstract}

Backgrounds Hepatocellular carcinoma ( $\mathrm{HCC})$ is the most frequent primary liver cancer and the third leading cause of cancer death. Krüppel-like factor 8 (KLF8) is an oncogene and has been shown playing an important role in $\mathrm{HCC}$, but the major involved signaling pathways are still unknown. Here, we systematically analyzed the role of KLF8 in HCC using RNA sequencing and the anti-H3K27 acetylation ChIP sequencing combined with bioinformatics analysis, and the results of data mining.

Results The results in this study showed that KLF8 worked as a transcription repressor in HCC and the main directly regulated ones were apoptosis-related genes. Furthermore, we verified the combination of KLF8 with some predicted target genes by ChIP, which supported the effectiveness of our analysis. Besides, we demonstrated that HMGA2 and MMP7, two predicted targets, were important participants in KLF8 mediated anti-apoptotic effect in HCC.

Conclusions Our work offers a panoramic view of KLF8's role in HCC for the first time and facilitates the discovery of new targets for HCC via KLF8.

\section{Background}

Hepatocellular carcinoma ( $\mathrm{HCC}$ ) is the most frequent primary liver cancer and is ranked as the sixth most common neoplasm and the third leading cause of cancer death, with an age-adjusted worldwide incidence of 10.1 cases per 100000 person-years in $2012^{1}$. Although significant progress regarding the diagnosis or treatment for $\mathrm{HCC}$ has been made in recent years, the exact mechanisms underlying its pathogenesis are still largely unknown ${ }^{2,3}$. Significantly, with the establishment and application of largescale sequencing and bioinformatics methods, it is possible to systematically elucidate the role of certain molecules in disease ${ }^{4,5}$. This will greatly enhance our in-depth understanding of complex diseases like HCC.

Krüppel-like factor 8 (KLF8) is one of KLF family whose members are all zinc finger transcription factors and control essential cellular processes including proliferation, differentiation and migration ${ }^{6-9}$. An excellent work in 2010 showed that KLF8 plays an important role in the pathogenesis of HCC ${ }^{10}$. The research demonstrated that KLF8 was positively correlated with the metastatic potential of HCC and promoted HCC proliferation and invasion both in vitro and in vivo. Our previous work also confirmed the positive correlation between KLF8 protein levels and HCC progression and proposed a reciprocal crosstalk between KLF8 and Wnt signaling pathway ${ }^{11}$. Besides, KLF8 has been reported aberrantly overexpressed in many other tumor types and various target genes of KLF8 have been identified ${ }^{12-15}$. However, most of the previous works focused on whether KLF8 participated in some known pathways and a systematic analysis is lacked, which impedes further application.

Here by deep sequencing combined with bioinformatics analysis, we systematically analyzed the role of KLF8 in HCC and exploited the major involved signaling pathways and possible target genes. 
Furthermore, we verified the predicted target genes by ChIP and selected HMGA2 and MMP7 for further analysis. HMGA2 and MMP7 were proved as important participants in KLF8 mediated anti-apoptotic effect in HCC. Our work helps set up a panoramic view of KLF8's role in HCC.

\section{Materials And Methods}

\section{Cell cultures}

Hepatocellular carcinoma cell lines LM3 and Huh-7 were purchased from The Institutes of Biomedical Sciences ( Fudan University, Shanghai, China) and The Cell Bank of Typical Culture Preservation Commission ( Chinese academy of sciences, Shanghai, China) respectively, and were cultured according to the supplier's instructions. The KLF8 ${ }^{\mathrm{KO}}{ }_{-L M 3}$ cell line was constructed by CRISPR/Cas 9 engineering ${ }^{16}$. The human KLF8 sequence was cloned and ligated into the GV303 plasmid (GeneChem, Shanghai). The titers of concentrated viral particles ranged between $5 \times 10^{8}$ and $1 \times 10^{9}$ transducing units $/ \mathrm{ml}$. Lentiviral particles were added to Huh -7 cells at a multiplicity of infection $(\mathrm{MOI})=20$, and the supernatant was changed $12 \mathrm{~h}$ after infection.

\section{RNA seq}

Total RNA was extracted from LM3 or KLF8 ${ }^{\mathrm{KO}}$-LM3 cells using TRIzol reagent (Invitrogen) according to the manufacturer's instructions. RNA quality was evaluated with a BioAnalyzer 2100 system (Agilent Technologies, USA). Small RNAs had linkers ligated to them and bar-coded cDNAs were prepared using a TruSeq Sample Prep Kit (Illumina, USA) following the manufacturer's instructions. Individual libraries were analyzed for the presence of linked cDNA at the appropriate size (140-150 bp) as determined by the BioAnalyzer. Subsequently, the amplified cDNA constructs were purified from agarose gel in preparation for sequencing analysis using the Illumina HiSeq 2500 platform (Illumina, CA, USA) according to the manufacturer's instructions at the Shanghai Biotechnology Corporation.

The RNA sequence reads were pre-processed using the FASTXToolkit to exclude low-quality reads (ambiguous $\mathrm{N}$, quality $<10 \mathrm{nt}$, and length $<18 \mathrm{nt}$ ) and $3^{\prime}$ adapter, $5^{\prime}$ adapter and poly(A) sequences). Further annotation analyses were performed using the commercial software CLC Genomic Workbench 5.5. After all annotation steps, the sequencing libraries were used for size distribution and saturation analysis.

\section{Western blot}

Primary cell cultures were homogenized in NP40 buffer (Beyotime, China) supplemented with PMSF (1 $\mathrm{mM}$, Sigma-Aldrich). The cell lysates were subjected to western blotting using the anti-caspase -3 (1:1000, 9662s, Cell Signaling Technology), anti-KLF8 (1:500, 2101276, Sigma-Aldrich), anti-Flag (1:1000, 
14793, Cell Signaling Technology) and HRP-conjugated anti-GAPDH (Kangcheng, Shanghai, China) antibodies. The protein bands were analyzed using Image Lab analysis (Bio-Rad).

\section{ChIP}

ChIP was performed using an EZ ChIP ${ }^{\mathrm{TM}}$ Chromatin Immunoprecipitation Kit (17-371, Millipore) following the manufacturer's protocol. Quantitative PCR was conducted to detect DNA fragments binding with KLF8. Primers were designed to detect predicted promoters or enhancers (Table S2). The ratio of DNA binding with KLF8 versus total DNA was calculated.

\section{Bioinformatics Analysis}

We employed MACS2 (https://pypi.python.org/pypi/MACS2) to identify H3K27ac peaks in both LM3 or KLF8 ${ }^{K O}$-LM3 cells. We set $\mathrm{H} 3 \mathrm{~K} 27 \mathrm{ac}$ in $\mathrm{KLF} 8^{\mathrm{KO}}-\mathrm{LM} 3$ cells as treatment and $\mathrm{H} 3 \mathrm{~K} 27 \mathrm{ac}$ in $\mathrm{LM} 3$ cells as control to detect $\mathrm{KLF}^{\mathrm{KO}}{ }_{-\mathrm{LM} 3}$ specific peaks ( $\mathrm{p}$-value was set as 0.00001 ). BETA was used to integrate differential expression calculated by Deseq2 (http://www.bioconductor.org) with KLF8 ${ }^{\mathrm{KO}}$-LM3 specific peaks (-d 2000-df 0.05) to obtain genes whose transcription levels are regulated by KLF8. We run FIMO (http://meme.sdsc.edu) over all of promoters for hits of KLF8 motif to further identify putative direct targets of KLF8 (Table S1).

\section{Apoptosis assay}

For apoptosis analysis, $\mathrm{LM} 3$ or $\mathrm{KLF}^{\mathrm{KO}}$-LM3 cells were stained with FITC-Annexin-V and PI using a Cell Apoptosis Analysis Kit (Sungene, Tianjin) according to the manufacturer's instructions. Annexin- $\mathrm{V}^{+} \mathrm{PI}^{-}$ cells were considered as cells in apoptosis.

\section{Cell proliferation assay}

Cell proliferation assay was carried out using CCK8 cell prolifetion assays kit (Dojindo, Japan) according to the manufacturer's instruction. Briefly, Huh-7 cells transfected with OE-KLF8 or OE-NC vectors or LM3/KLF8 ${ }^{\mathrm{KO}}$-LM3 cells were placed into 96 -well plate. After $18 \mathrm{~h}$ after adding $20 \mathrm{uM}$ camptothecin (sigmaaldrich), $10 \mu \mathrm{lCCK}-8$ assay solution was added. Then, after incubation for another $1 \mathrm{~h}$, optical density (OD) at $450 \mathrm{~nm}$ was measured with an enzyme immunoassay analyzer (Thermo Fisher Scientific, Inc., Waltham, MA, USA) to estimate cell proliferation.

\section{Statistical analysis}


A two-tailed Student's $t$-test was applied for statistical comparison of 2 groups. The data are presented as the mean \pm SD. unless otherwise indicated and "mean \pm SD" generally represents biological replicates. A $p<0.05$ was considered statistically significant.

\section{Results}

\section{Knockout of KLF8 in the LM3 cell line promoted cell apoptosis via up-regulating the expression of apoptosis- related genes}

To elucidate the role of KLF8 on the gene expression pattern in HCC, we selected the LM3 cell line in which KLF8 is highly expressed for study. Meanwhile, we constructed a KLF8 knockout LM3 cell line (KLF8 ${ }^{\mathrm{KO}}$-LM3) using CRISPR/Cas9 technology (Fig. S1A). RNA sequencing was employed and the expression of 1816 genes were changed between the two cell lines (Fig. 1A), indicating that KLF8 possessed a general and critical influence on the gene expression in LM3. Further gene ontology (GO) analysis showed that the genes up-regulated after KLF8 knockout were mainly apoptosis-related genes (Fig. 1B), while the down-regulated ones were associated with development processes of tissues or organs (Fig. 1C). Pathway analysis (KEGG) of differentially expressed genes was also conducted, but no HCC pathways were found, indicating that KLF8 was not specifically involved in HCC (Fig. 1D, E). The RNA sequencing results showed that knockout of KLF8 in LM3 cell line mainly promoted the expression of apoptosis-related genes, so we conducted the flow cytometry analysis of Annexin $\mathrm{V}$ and PI. The results showed that KLF8 did significantly affect the apoptosis of HCC (Fig. 1F, G). The CCK8 experiments showed a consistent result (Fig. S2). These results showed that KLF8 probably worked as a repressor of apoptosis-related genes in HCC.

\section{Knockout of KLF8 changed the acetylation of H3K27 in the LM3 cell line}

As a transcription factor, KLF8 could regulate gene expression through activating or inhibiting RNA transcription directly, but genes differentially expressed in RNA sequencing may be influenced in an indirect way, so a more accurate analysis is needed. However, owing to the lack of qualified anti-KLF8 antibody, we failed to conduct ChIP sequencing. The process that a transcription factor regulates gene expression is often accompanied with histone modification of related enhancers or promoters, among which the one positive correlated with transcription, $\mathrm{H} 3 \mathrm{~K} 27$ acetylation, is a typical indicator ${ }^{17-20}$. To find genes whose transcription probably directly regulated by KLF8, we performed the ChIP sequencing assay using the anti-H3K27 acetylation antibody, which bypasses the need for KLF8 antibody with ChIP efficiency. We obtained the H3K27 acetylation data with high quality in both $\mathrm{LM} 3$ and KLF8 ${ }^{\mathrm{KO}}{ }_{-\mathrm{LM}} \mathrm{B}$ cell lines (Fig. 2A). The comparison of H3K27 acetylation between LM3 and KLF8 ${ }^{\mathrm{KO}}$-LM3 cell lines showed that knockout of KLF8 leaded to a much higher H3K27 acetylation level, showing that KLF8 worked as a 
repressor of gene transcription in HCC (Fig. 2B). However, because of the huge background transcriptional activity, GO analysis of $\mathrm{H} 3 \mathrm{~K} 27$ acetylation genes showed no significant difference in the major functional subgroups between $\mathrm{LM} 3$ and $\mathrm{KLF}^{\mathrm{KO}}$-LM3 cell lines (Fig. 2C, D). Pathway analysis also showed no significant difference between the two groups (Fig. 2E, F). These results indicated that KLF8 worked as a transcription repressor in HCC, although the major biological processes were not affected.

\section{Knockout of KLF8 in the LM3 cell line increased the level of H3K27 acetylation of apoptosis-related genes}

Although knockout of KLF8 failed to affect the H3K27 acetylation of a certain gene subtype in LM3 cells, a combined analysis of the RNA and the anti-H3K27 acetylation ChIP sequencing probably helped us. Analysis by integration of transcriptome and ChIP-seq data with "BETA" 21 showed that up-regulated genes were more likely the targets of KLF8 (Fig. 3A). We next screened out genes acetylated as well as highly expressed in the KLF8 ${ }^{\mathrm{KO}}$-LM3 cell line (Table S1). 532 genes were found and the "peak score" of H3K27 acetylation were calculated (Fig. 3B). GO analysis showed that these genes were mainly apoptosis-related genes (Fig. 3C). KEGG showed no special pathways involved in tumor growth or migration existed (Fig. 3D). This conjoint analysis confirmed the accuracy of the RNA sequencing. It also indicated that the role of KLF8 in HCC may be implemented by regulating the transcription of certain apoptosis-related genes.

\section{KLF8 directly regulated apoptosis-related genes}

The combined analysis of the transcriptome and the anti-H3K27 acetylation ChIP sequencing filtered out 532 genes may be regulated in the $\mathrm{KLF}^{\mathrm{KO}}-\mathrm{LM} 3$ cell line. We then analyzed the promoters of these genes and the KLF8 recognition motif was found in 510 ones (Fig. 4A, B; Table S1). The results indicated that anti-H3K27 acetylation ChIP sequencing was a reliable alternative method while KLF8 ChIP sequencing data was unattainable. Next, we focused on the apoptosis-related genes which were probably the key participants in KLF8-mediated effects in HCC. After excluding the genes whose roles in HCC have been well-established and the ones with few motifs, ChIP experiments were conducted to verify the direct regulation and the specific binding between KLF8 and several apoptosis-related genes were confirmed (Fig. 4C-E; Table S2). These results showed that KLF8 directly regulated the expression of apoptosisrelated genes.

\section{HMGA2 and MMP7 participated in the anti-apoptotic effect mediated by KLF8}

Further, we intended to select molecules for functional verification from the apoptosis-related genes directly regulated by KLF8. HMGA2 and MMP7 exhibited significant changes in gene expression and 
concordant changes in H3K27ac levels over promoter regions upon KLF8 knockout (Fig. 5A, B). HMGA2 was reported participating in the proliferation of many cancer types ${ }^{22-24}$, and MMP7 was found affecting the apoptotic process mediated by FAS/FASL system as well as $\mathrm{N}$-cadherin ${ }^{25,26}$. However, their roles in HCC's apoptosis were not clear. Apoptotic cells were quantified using flow cytometry after Annexin V-PI staining, and results showed that both HMGA2 and MMP7 affected KLF8-mediated antiapoptotic effects (Fig. 5C, D). Detection of caspase 3 activity using western blot revealed similar results (Fig. 5E, F). These results indicate that HMGA2 and MMP7 participate in KLF8-mediated anti-apoptotic effect in HCC, which supported the reliability of our former analysis.

\section{Discussion}

As an important oncogene, KLF8 has drawn a lot of attention in the field of malignant tumors including HCC in recent years. However, a systematic analysis of molecules regulated by KLF8 was lacked. Here, we analyzed the genes regulated by KLF8 in HCC using large-scale sequencing and bioinformatics analysis, and found that KLF8 mainly regulated apoptosis-related genes directly. We also found new pro-apoptotic genes in HCC, showing the application value of the strategy. Unfortunately, the quality of anti-KLF8 ChIP sequencing data was poor owing to low antibody quality, and the promoters regulated by KLF8 were analyzed by H3K27 acetylation and motif analysis indirectly. This deficiency is expected to be made up in the future.

KLF8 was first identified as a CACCC-box binding protein that associates with CtBP and represses transcription in $2000^{12}$ and the classical regulation mechanism of KLF8 was as a downstream target of focal adhesion kinase and an upstream regulator of cyclin D1 ${ }^{13}$. Besides, KLF8 was also found inducing epithelial to mesenchymal transition and epithelial cell invasion via directly bound and repressed the promoter of E-cadherin ${ }^{14}$. Poly (ADP-ribose) polymerase-1 (PARP-1) was also recognized as a KLF8interacting and -regulating protein ${ }^{27}$. A reciprocal crosstalk between KLF8 and Wnt signal pathway was proposed in our previous work ${ }^{11}$. However, these fragmented findings make the role of KLF8 in a certain situation complicated to evaluate. We aimed to achieve a comprehensive understanding of KLF8 in HCC, and fortunately the major role was quite obvious owing to the advanced methods. This strategy helps achieve an in-depth and comprehensive understanding of a certain molecule in defined conditions, which is very important for a potential target.

HMGA2 is a member of the HMGA family which is an important component of DNA architecture 28,29 . HMGA2 participates in many biological processes including the apoptotic pathway ${ }^{30}$. HMGA2 has been found overexpressed in many malignant tissues and is often considered as an oncogene ${ }^{31-34}$, but few studies focused on the role of HMGA2 in HCC except for ones considering it as an oncogene on assumptions ${ }^{35-37}$. Our work shed a light on the complicated roles of HMGA2 in HCC. MMP7 is a secreted protease and a complicated participant in apoptosis. It has been reported inhibiting cell death in colon cancer cells while promoting smooth muscle cell apoptosis ${ }^{26,38}$. Here, MMP7 has been shown 
promote apoptosis in HCC, but the detailed mechanism is still unknown. More work needs to be done to make the two apoptosis-related genes qualified targets for the treatment of HCC.

\section{Conclusion}

In summary, our work offered a panoramic view of KLF8's role in HCC for the first time, and found that KLF8 mainly regulated apoptosis-related genes directly. Our work helps establish an in-depth insight of KLF8's role in HCC and facilitates the discovery of new targets for HCC via KLF8.

\section{Notes}

Hao Xingand Jin Zhang contributed equally to this work.

\section{Abbreviations}

HCC: Hepatocellular carcinoma; KLF8: Krüppel-like factor 8; CRISPR: clustered regularly interspaced short palindromic repeat; $\mathrm{MOI}$ : multiplicity of infection; GAPDH: glyceraldehyde phosphate dehydrogenase; ChIP: Chromatin Immunoprecipitation; OD: optical density; SD: Standard deviation; KEGG: Kyoto Encyclopedia of Genes and Genomes; GO: Gene Ontology; HMGA2: high-mobility group AT-hook 2; MMP: matrix metallopeptidase; FAS: factor associated suicide; CtBP: C-terminal binding protein; PARP-1: Poly (ADP-ribose) polymerase-1.

\section{Declarations}

\section{Ethics approval and consent to participate}

Not applicable.

\section{Consent for publication}

Not applicable.

\section{Availability of data and material}

The data is available through request to authors.

\section{Competing interests}

The authors declare that they have no competing interests. 


\section{Funding}

This work was supported by National Natural Science Foundation of China (81472284 and 81672699 ), Shanghai Pujiang Program (16PJD004), and Charitable Project on Scientific Research of Shanghai.

\section{Authors' contributions}

FS and TY designed the study, HX, JZ and MCW analyzed the sequencing data. MDW, JH and TY participated in data analysis. HX, JH, CL, ZLL, HW, LL, MCW, FS and TY conducted experimental studies, $\mathrm{HX}, \mathrm{KLZ}$ and TY wrote and revised the manuscript. All authors have read and approved the manuscript.

\section{Acknowledgements}

Not applicable.

\section{References}

1.Forner A, Reig M, Bruix J. Hepatocellular carcinoma. Lancet 2018. DOI: 10.1016/S01406736(18)30010-2

2.Fu J, Wang H. Precision diagnosis and treatment of liver cancer in China. Cancer letters 2018; 412: 283-288. DOI: 10.1016/j.canlet.2017.10.008

3.Zhu H, Wu J, Shen X. Genome-wide association study: new genetic insights into HBV/HCV-related hepatocellular carcinoma genomes. Scandinavian journal of gastroenterology 2017; 52: 209-215. DOI: $10.1080 / 00365521.2016 .1245778$

4.Sudhagar A, Kumar G, El-Matbouli M. Transcriptome Analysis Based on RNA-Seq in Understanding Pathogenic Mechanisms of Diseases and the Immune System of Fish: A Comprehensive Review. International journal of molecular sciences 2018; 19. DOI: 10.3390/ijms19010245

5.Milan T, Wilhelm BT. Mining Cancer Transcriptomes: Bioinformatic Tools and the Remaining Challenges. Molecular diagnosis \& therapy 2017; 21:249-258. DOI: 10.1007/s40291-017-0264-1

6.McConnell BB, Yang VW. Mammalian Kruppel-like factors in health and diseases. Physiological reviews 2010; 90:1337-1381. DOI: 10.1152/physrev.00058.2009

7.Kim CK, He P, Bialkowska AB, Yang VW. SP and KLF Transcription Factors in Digestive Physiology and Diseases. Gastroenterology 2017; 152: 1845-1875. DOI: 10.1053/j.gastro.2017.03.035

8.Cao Z, Sun X, Icli B, Wara AK, Feinberg MW. Role of Kruppel-like factors in leukocyte development, function, and disease. Blood 2010; 116: 4404-4414. DOI: 10.1182/blood-2010-05-285353 
9.Lu XJ, Shi Y, Chen JL, Ma S. Kruppel-like factors in hepatocellular carcinoma. Tumour biology: the journal of the International Society for Oncodevelopmental Biology and Medicine 2015; 36: 533-541. DOI: $10.1007 /$ s13277-015-3127-6

10.Li JC, Yang XR, Sun HX, Xu Y, Zhou J, Qiu SJ, Ke AW, Cui YH, Wang ZJ, Wang WM, Liu KD, Fan J. Upregulation of Kruppel-like factor 8 promotes tumor invasion and indicates poor prognosis for hepatocellular carcinoma. Gastroenterology 2010; 139: 2146-2157 e2112. DOI:

10.1053/j.gastro.2010.08.004

11.Yang T, Cai SY, Zhang J, Lu JH, Lin C, Zhai J, Wu MC, Shen F. Kruppel-like factor 8 is a new Wnt/betacatenin signaling target gene and regulator in hepatocellular carcinoma. PloS one 2012; 7: e39668. DOI: 10.1371/journal.pone.0039668

12.van Vliet J, Turner J, Crossley M. Human Kruppel-like factor 8: a CACCC-box binding protein that associates with CtBP and represses transcription. Nucleic acids research 2000; 28: 1955-1962.

13.Zhao J, Bian ZC, Yee K, Chen BP, Chien S, Guan JL. Identification of transcription factor KLF8 as a downstream target of focal adhesion kinase in its regulation of cyclin D1 and cell cycle progression. Molecular cell 2003; 11:1503-1515.

14.Wang X, Zheng M, Liu G, Xia W, McKeown-Longo PJ, Hung MC, Zhao J. Kruppel-like factor 8 induces epithelial to mesenchymal transition and epithelial cell invasion. Cancer research 2007; 67: 7184-7193. DOI: 10.1158/0008-5472.CAN-06-4729

15.Lahiri SK, Zhao J. Kruppel-like factor 8 emerges as an important regulator of cancer. American journal of translational research 2012; 4: 357-363.

16.Cho SW, Kim S, Kim JM, Kim JS. Targeted genome engineering in human cells with the Cas9 RNAguided endonuclease. Nature biotechnology 2013; 31:230-232. DOI: 10.1038/nbt.2507

17.Turner BM. Histone acetylation and control of gene expression. Journal of cell science 1991; 99 (Pt 1): 13-20.

18.Smith E, Shilatifard A. Enhancer biology and enhanceropathies. Nature structural \& molecular biology 2014; 21: 210-219. DOI: 10.1038/nsmb.2784

19.Chen Y, Jorgensen M, Kolde R, Zhao X, Parker B, Valen E, Wen J, Sandelin A. Prediction of RNA Polymerase II recruitment, elongation and stalling from histone modification data. BMC genomics 2011; 12: 544. DOI: 10.1186/1471-2164-12-544

20.Roe JS, Hwang Cl, Somerville TDD, Milazzo JP, Lee EJ, Da Silva B, Maiorino L, Tiriac H, Young CM, Miyabayashi K, Filippini D, Creighton B, Burkhart RA, Buscaglia JM, Kim EJ, Grem JL, Lazenby AJ, Grunkemeyer JA, Hollingsworth MA, Grandgenett PM, Egeblad M, Park Y, Tuveson DA, Vakoc CR. 
Enhancer Reprogramming Promotes Pancreatic Cancer Metastasis. Cell 2017; 170: 875-888 e820. DOI: 10.1016/j.cell.2017.07.007

21.Wang S, Sun H, Ma J, Zang C, Wang C, Wang J, Tang Q, Meyer CA, Zhang Y, Liu XS. Target analysis by integration of transcriptome and ChIP-seq data with BETA. Nature protocols 2013; 8: 2502-2515. DOI: $10.1038 /$ nprot. 2013.150

22.Fedele M, Palmieri D, Fusco A. HMGA2: A pituitary tumour subtype-specific oncogene? Molecular and cellular endocrinology 2010; 326:19-24. DOI: 10.1016/j.mce.2010.03.019

23.Kaur H, Ali SZ, Huey L, Hutt-Cabezas M, Taylor I, Mao XG, Weingart M, Chu Q, Rodriguez FJ, Eberhart $\mathrm{CG}$, Raabe EH. The transcriptional modulator HMGA2 promotes stemness and tumorigenicity in glioblastoma. Cancer letters 2016; 377: 55-64. DOI: 10.1016/j.canlet.2016.04.020

24.Wu J, Zhang S, Shan J, Hu Z, Liu X, Chen L, Ren X, Yao L, Sheng H, Li L, Ann D, Yen Y, Wang J, Wang X. Elevated HMGA2 expression is associated with cancer aggressiveness and predicts poor outcome in breast cancer. Cancer letters 2016; 376: 284-292. DOI: 10.1016/j.canlet.2016.04.005

25.Poulaki V, Mitsiades CS, Mitsiades N. The role of Fas and FasL as mediators of anticancer chemotherapy. Drug resistance updates: reviews and commentaries in antimicrobial and anticancer chemotherapy 2001; 4: 233-242. DOI: 10.1054/drup.2001.0210

26.Williams H, Johnson JL, Jackson CL, White SJ, George SJ. MMP-7 mediates cleavage of N-cadherin and promotes smooth muscle cell apoptosis. Cardiovascular research 2010; 87: 137-146. DOI: $10.1093 / \mathrm{cvr} / \mathrm{cvq0} 2$

27.Lu H, Wang X, Li T, Urvalek AM, Yu L, Li J, Zhu J, Lin Q, Peng X, Zhao J. Identification of poly (ADPribose) polymerase-1 (PARP-1) as a novel Kruppel-like factor 8-interacting and -regulating protein. The Journal of biological chemistry 2011; 286: 20335-20344. DOI: 10.1074/jbc.M110.215632

28.Cleynen I, Van de Ven WJ. The HMGA proteins: a myriad of functions (Review). International journal of oncology 2008; 32: 289-305.

29.Reeves R. High mobility group (HMG) proteins: Modulators of chromatin structure and DNA repair in mammalian cells. DNA repair 2015; 36: 122-136. DOI: 10.1016/j.dnarep.2015.09.015

30.Fujikane R, Komori K, Sekiguchi M, Hidaka M. Function of high-mobility group A proteins in the DNA damage signaling for the induction of apoptosis. Scientific reports 2016; 6:31714. DOI:

10.1038/srep31714

31.Gao X, Dai M, Li Q, Wang Z, Lu Y, Song Z. HMGA2 regulates lung cancer proliferation and metastasis. Thoracic cancer 2017; 8: 501-510. DOI: 10.1111/1759-7714.12476 
32.Esmailzadeh S, Mansoori B, Mohammadi A, Shanehbandi D, Baradaran B. siRNA-Mediated Silencing of HMGA2 Induces Apoptosis and Cell Cycle Arrest in Human Colorectal Carcinoma. Journal of gastrointestinal cancer 2017; 48: 156-163. DOI: 10.1007/s12029-016-9871-z

33.Zhang H, Tang Z, Deng C, He Y, Wu F, Liu O, Hu C. HMGA2 is associated with the aggressiveness of tongue squamous cell carcinoma. Oral diseases 2017; 23: 255-264. DOI: 10.1111/odi.12608

34.Cai J, Shen G, Liu S, Meng Q. Downregulation of HMGA2 inhibits cellular proliferation and invasion, improves cellular apoptosis in prostate cancer. Tumour biology: the journal of the International Society for Oncodevelopmental Biology and Medicine 2016; 37: 699-707. DOI: 10.1007/s13277-015-3853-9

35.Huang W, Li J, Guo X, Zhao Y, Yuan X. miR-663a inhibits hepatocellular carcinoma cell proliferation and invasion by targeting HMGA2. Biomedicine \& pharmacotherapy = Biomedecine \& pharmacotherapie 2016; 81: 431-438. DOI: 10.1016/j.biopha.2016.04.034

36.Ou W, Lv J, Zou X, Yao Y, Wu J, Yang J, Wang Z, Ma Y. Propofol inhibits hepatocellular carcinoma growth and invasion through the HMGA2-mediated Wnt/beta-catenin pathway. Experimental and therapeutic medicine 2017; 13: 2501-2506. DOI: 10.3892/etm.2017.4253

37.Wang Y, Chen F, Zhao M, Yang Z, Li J, Zhang S, Zhang W, Ye L, Zhang X. The long noncoding RNA HULC promotes liver cancer by increasing the expression of the HMGA2 oncogene via sequestration of the microRNA-186. The Journal of biological chemistry 2017; 292: 15395-15407. DOI:

10.1074/jbc.M117.783738

38.Zhang W, Li Y, Yang L, Zhou B, Chen KL, Meng WJ, Liu Y, Hu JK, Sun XF, Zhou ZG. Knockdown of MMP-7 inhibits cell proliferation and enhances sensitivity to 5-Fluorouracil and X-ray irradiation in colon cancer cells. Clinical and experimental medicine 2014; 14: 99-106. DOI: 10.1007/s10238-012-0212-7

\section{Figures}


A

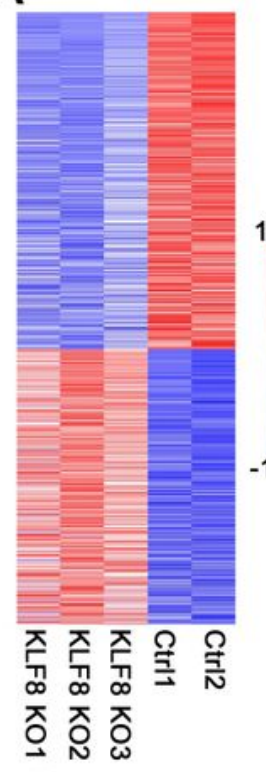

D

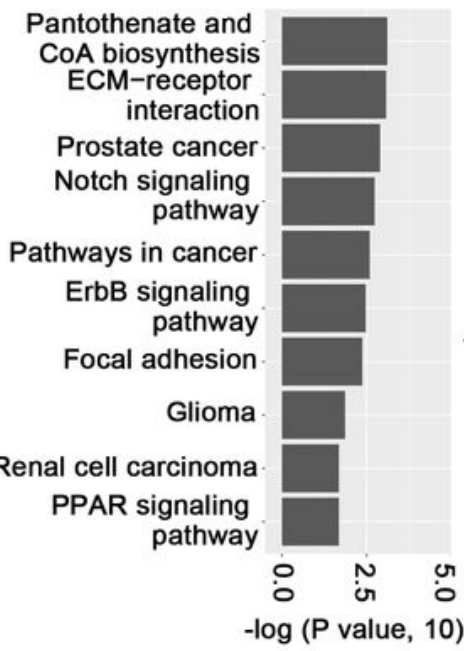

B

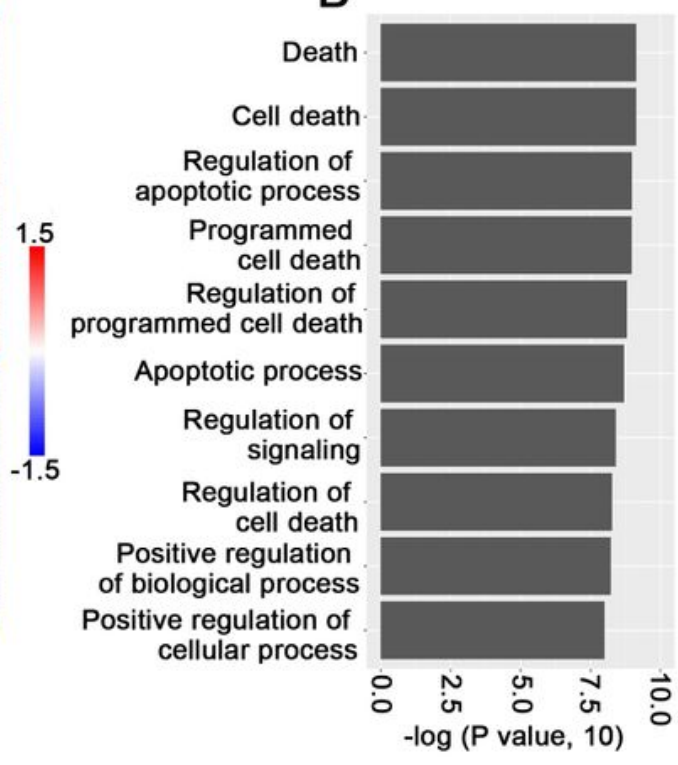

E

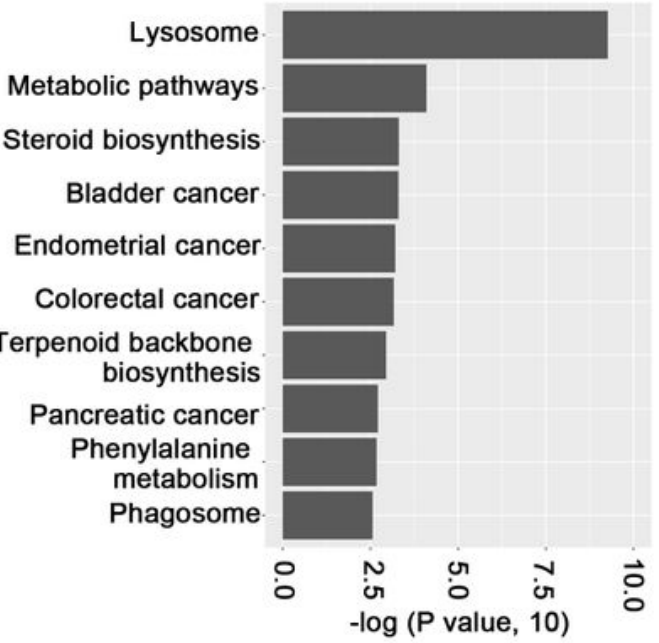

C

Anatomical structure development System development

Tissue development

Developmental process

Single-organism developmental process

Cellular response to chemical stimulus Response to organic substance Localization

Anatomical structure morphogenesis

Multicellular organismal development

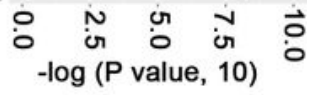

G

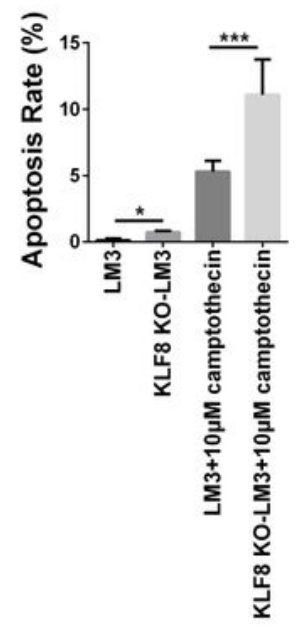

F

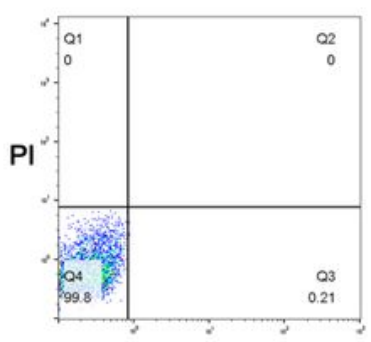

FITC-Annexin-V

LM3

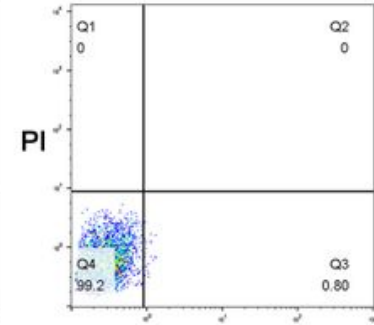

FITC-Annexin-V

KLF8 KO-LM3

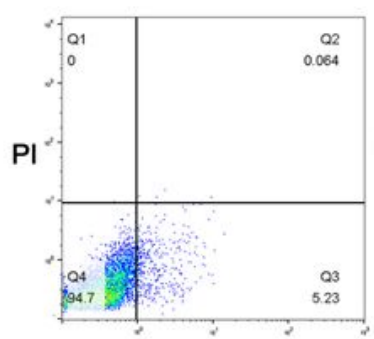

FITC-Annexin-V

LM3+10uM camptothecin

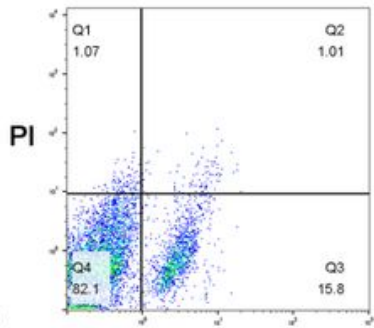

FITC-Annexin-V

KLF8 KO-LM3+ 10uM camptothecin

Figure 1

Knockout of KLF8 promoted the expression of apoptosis-related genes A. Hierarchical clustering analysis of genes that were differentially expressed between KLF8KO-LM3 cells and control (greater than 1.5-fold; $p<0.05)$. Expression values were represented in shades of red and green, which indicated expression above and below the median expression value across all samples (log scale 2, from 1.5 to -1.5), respectively. B,C . GO analysis of genes up-regulated (B) or down-regulated (C) in KLF8KO-LM3 cells. D,E. 
KEGG analysis of genes up-regulated (D) or down-regulated (E) in KLF8KO-LM3 cells. F. Representative flow cytometry analysis after Annexin-V/PI staining in LM3 or KLF8KO-LM3 cells. G. Apoptosis rate of LM3 or KLF8KO-LM3 cells in (F). N = 3 experiments.

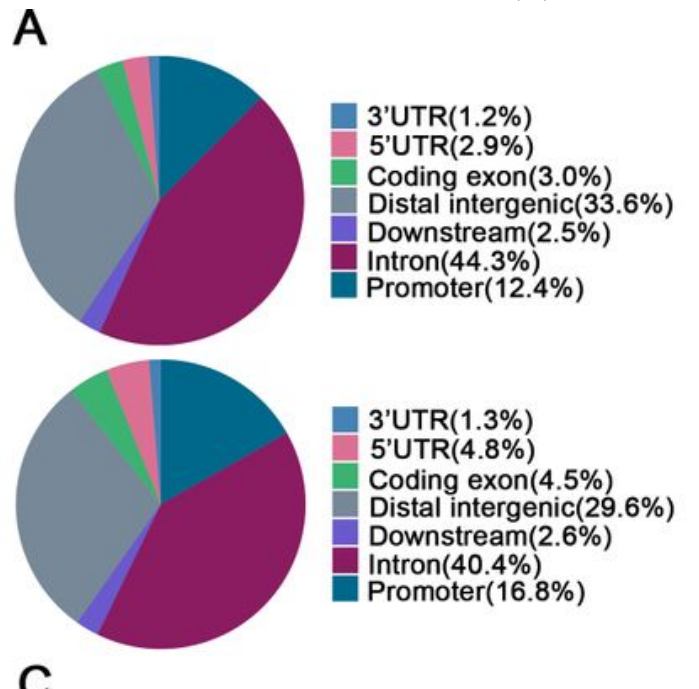

C

Cellular metabolic process Cellular macromolecule metabolic process

Organelle organization

Metabolic process

Cellular nitrogen compound metabolic process

Primary metabolic process

Nitrogen compound metabolic process Organic substance metabolic process

Macromole culemetabolic process

Nucleic acid metabolic process

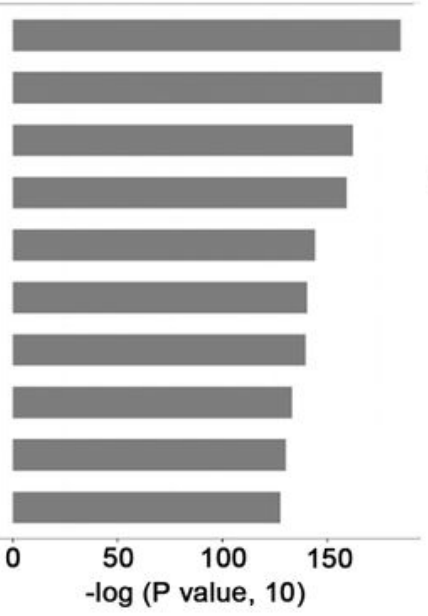

E

Protein processing in endoplasmic reticulum

spliceosome

Cell cycle

Ubiquitin mediated proteolysis

RNA transport

Ribosome

Huntington's disease

Metabolic pathway

Pyrimidine metabolism

RNA degradation

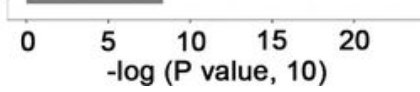

B

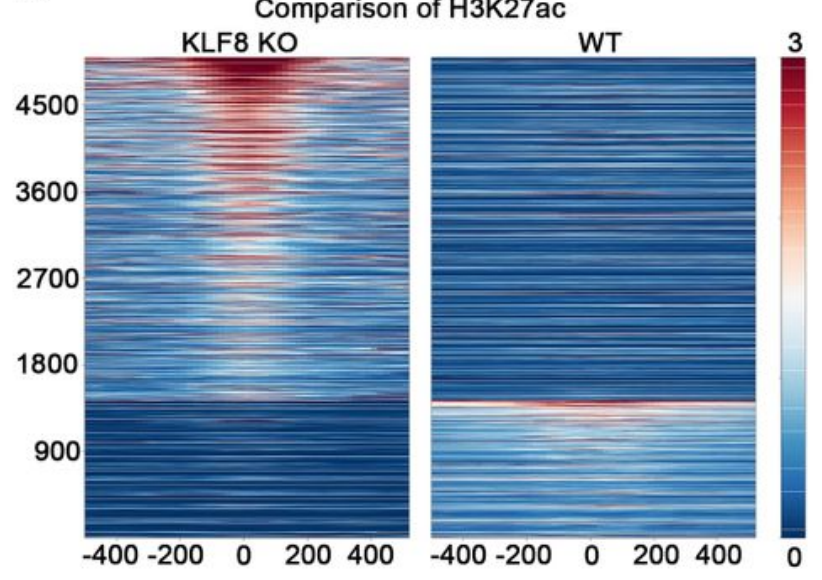

D

Cellular metabolic process Cellular macromolecule metabolic process Metabolic process

Cellular nitrogen compound metabolic process

Organelle organization

Primary metabolic process Nitrogen compound metabolic process Organic substance metabolic process Macromole culemetabolic process

Nucleic acid metabolic process

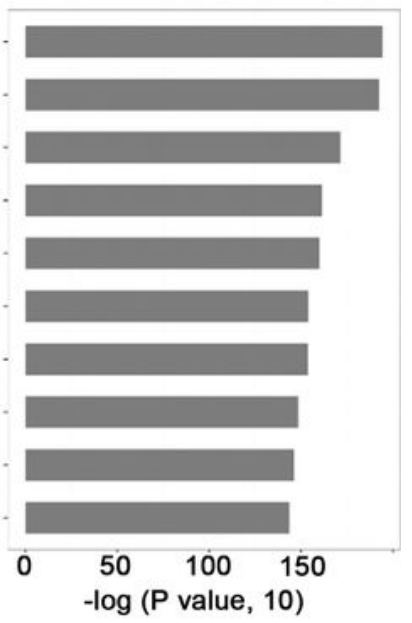

F

Protein processing in endoplasmic reticulum spliceosome Cell cycle Ubiquitin mediated proteolysis Ribosome RNA transport Endocytosis

Pyrimidine metabolism Huntington's disease Chronic myeloid leukemia

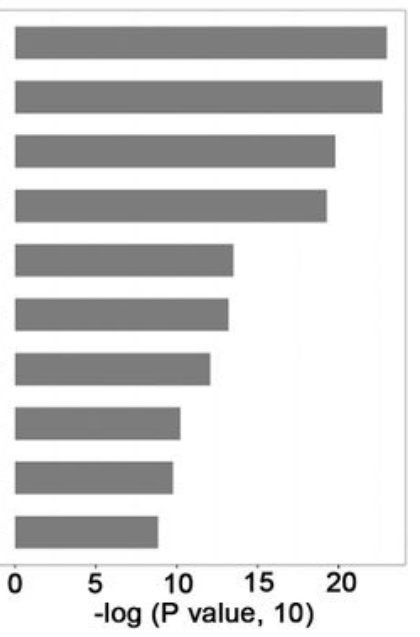

Figure 2

Knockout of KLF8 changed the H3K27 acetylation genes in the LM3 cell line A. Distribution of DNA fragments obtained by anti-H3K27ac ChIP in KLF8KO-LM3 (up) or LM3 (down) cells. B. Comparison of 
H3K27 acetylation between KLF8KO-LM3 (left) and LM3 (right) cells. H3K27ac ChIP-seq signal are plotted on center-aligned peaks from either KLF8KO-LM3 or LM3. K-Means cluster is employed to separate peaks with more enrichment in KLF8KO-LM3 and those with more enrichment in LM3. The significant enrichment of H3K27ac upon KLF8 KO suggests repressive role of KLF8 in gene regulation. C,D . GO analysis of H3K27 acetylation genes in KLF8KO-LM3 (C) or LM3 (D) cells. E,F . KEGG analysis of H3K27 acetylation genes in KLF8KO-LM3 (E) or LM3 (F) cells.

A

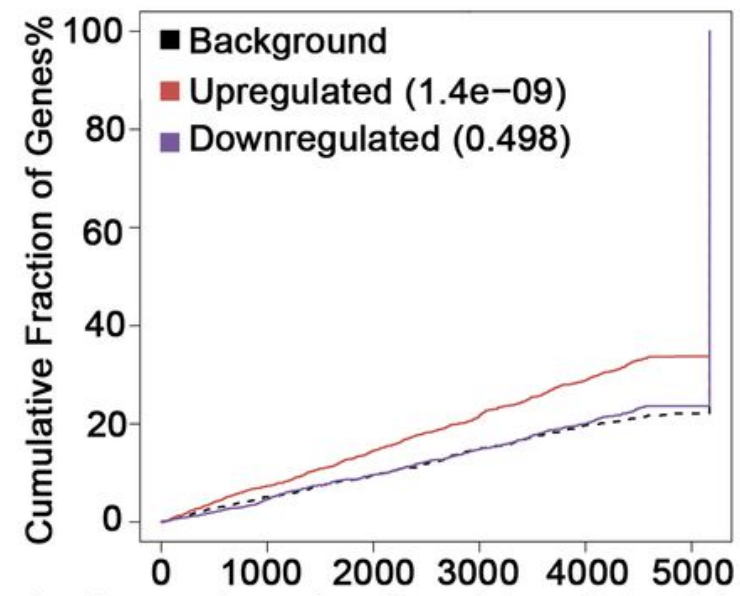

Rank of genes based on Regulatory Potential Score (from high to low)

\section{C}

Regulation of programmed cell death

Mitotic cell cycle

Regulation of cell death

Regulation of apoptotic process
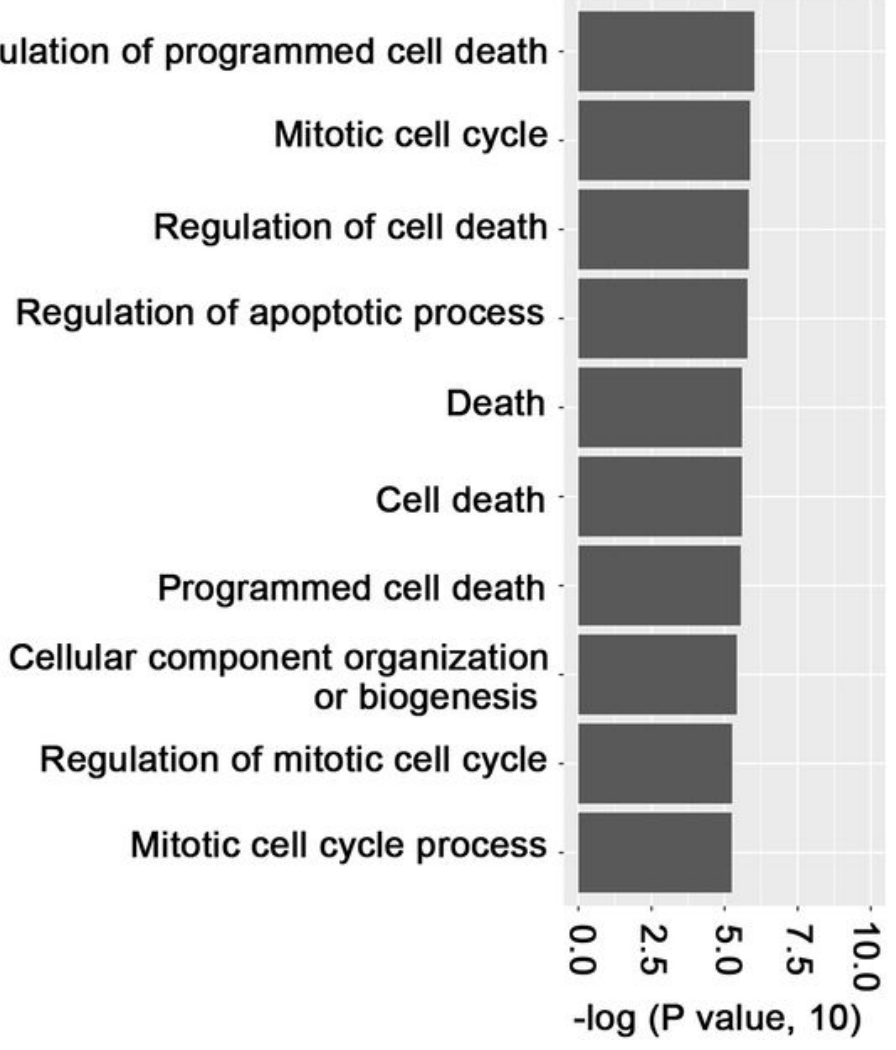
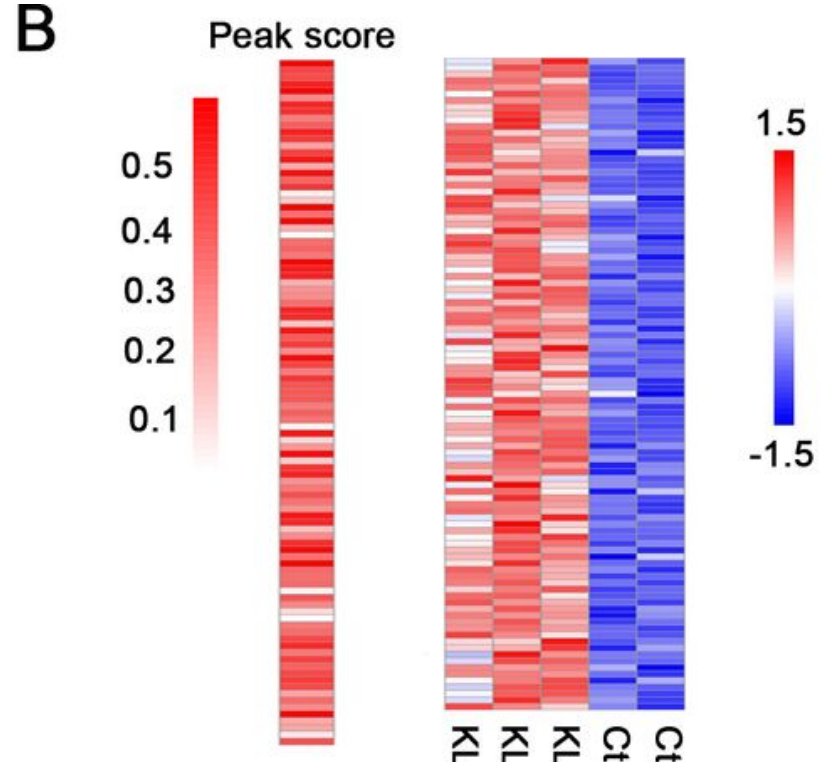

D

Protein processing in endoplasmic reticulum

Nicotinate and nicotinamide metabolism

Proteasome

Pyrimidine metabolism

Ribosome biogenesis in eukaryotes

Metabolic pathway

Focal adhesion

ECM-receptor interaction

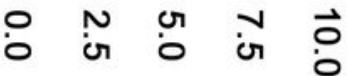

$$
\begin{aligned}
& \text {-log (P value, 10) }
\end{aligned}
$$

Figure 3 
Knockout of KLF8 in the LM3 cell line increased the level of H3K27 acetylation of apoptosis-related genes A. BETA activating/repressive function prediction of the transcriptome and H3K27 acetylation data from KLF8KO-LM3 or LM3 cells. The red and the purple lines represent the upregulated and downregulated genes, respectively. The dashed line indicates the nondifferentially expressed genes as background. Genes are cumulated by the rank on the basis of the regulatory potential score from high to low. P values that represent the significance of the UP or DOWN group distributions in the brackets are compared with the NON group by the Kolmogorov-Smirnov test. B. Left, the H3K27 acetylation peak score of the top 100 genes differentially expressed as well as H3K27 acetylation changed between KLF8KO-LM3 cells and control. Right, the hierarchical clustering of the top 100 genes in left. C. GO analysis of the overlapped genes (differentially expressed as well as H3K27 acetylation changed). D. KEGG analysis of the overlapped genes.
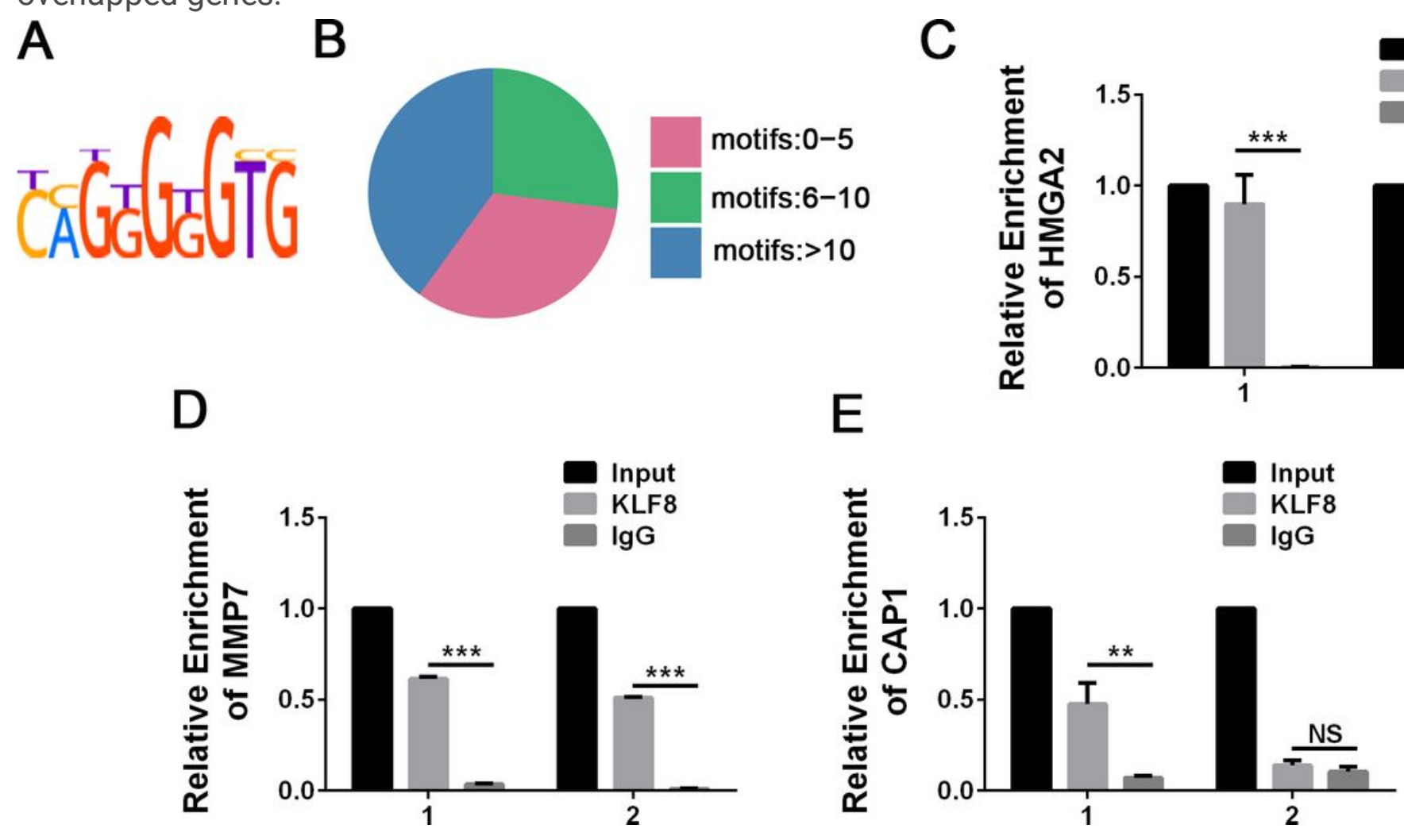

\section{Figure 4}

KLF8 directly regulated apoptosis-related genes A. A schematic map of the motif recognized by KLF8. B. Distribution of the number of KLF8 motifs in the overlapped genes (differentially expressed as well as H3K27 acetylation changed). C-E. ChIP analysis of overlapped apoptosis-related genes (C, HMGA2; D, MMP7; E, CAP1) with an anti-KLF8 antibody, $n=3$ experiments. 
A

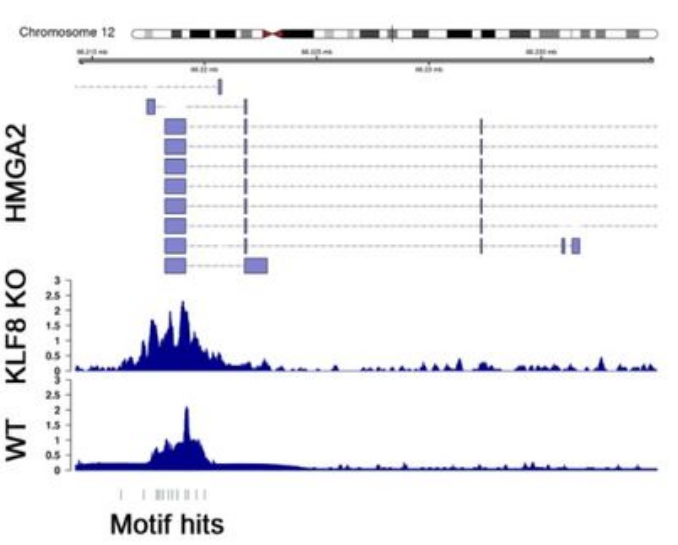

C

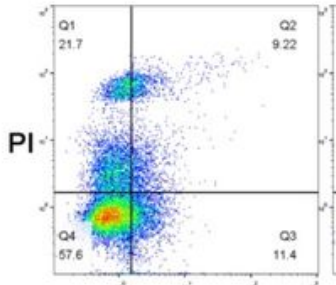

FITC-Annexin-V LM3+Ctrli

D

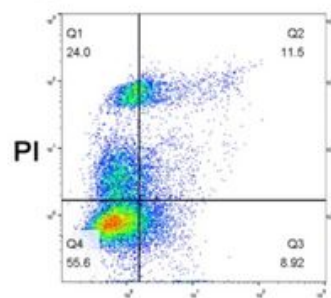

FITC-Annexin-V

LM3+Ctrli

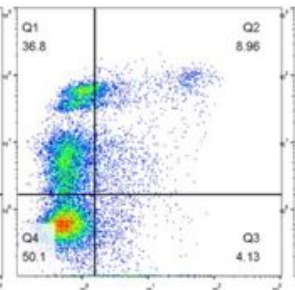
LM3+HMGA2i
FITC-Annexin-V

FITC-Annexin-V
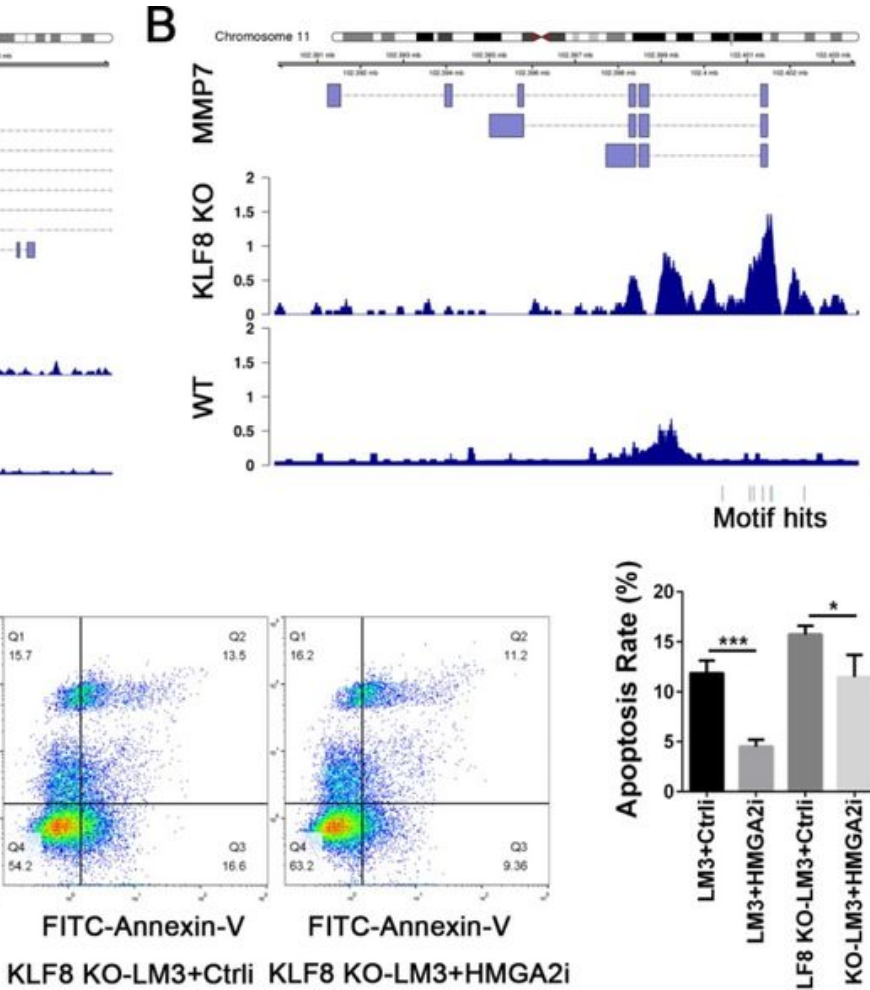

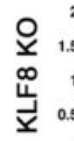

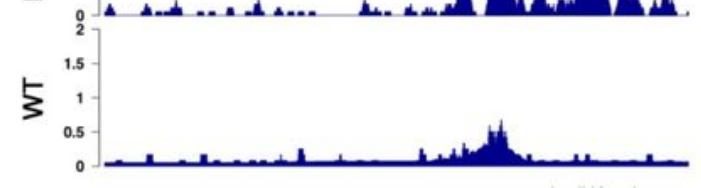

Motif hits
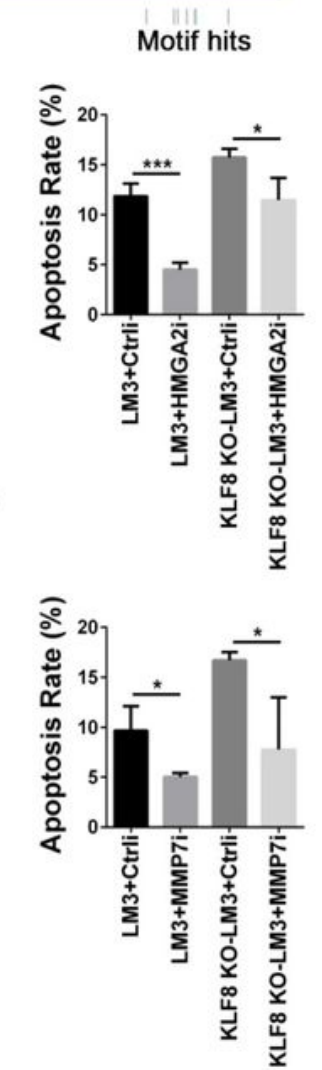

E

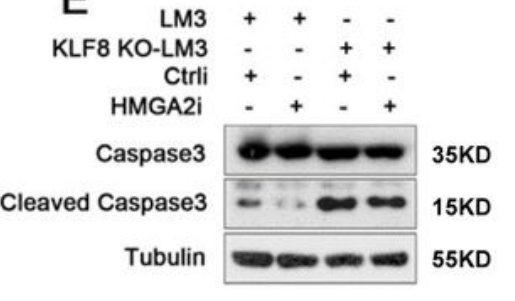

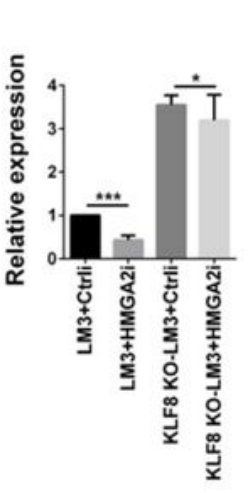

$\mathrm{F}$

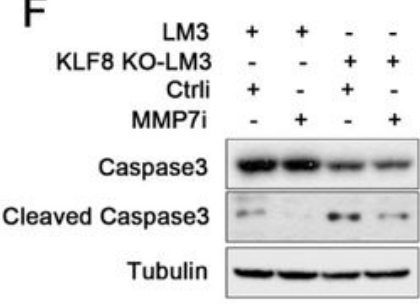

FITC-Annexin-V

FITC-Annexin-V

LM3+MMP7i

KLF8 KO-LM3+Ctrli KLF8 KO-LM3+MMP7i

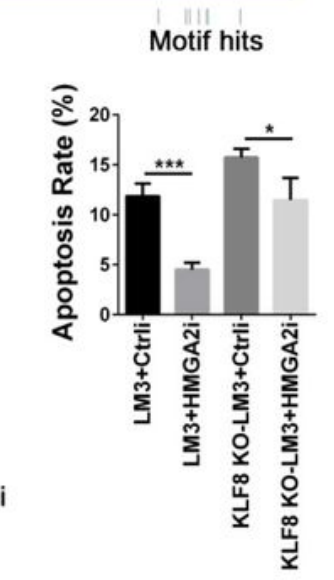


siRNA or control siRNA, $n=3$ experiments. E. Western blot analysis of activated caspase 3 in LM3 or KLF8KO-LM3 cells transfected with HMGA2 siRNA or control siRNA, $n=3$ experiments. F. Western blot analysis of activated caspase 3 in LM3 or KLF8KO-LM3 cells transfected with MMP7 siRNA or control siRNA, $n=3$ experiments.

\section{Supplementary Files}

This is a list of supplementary files associated with this preprint. Click to download.

- Supplementaltable1.Genesdifferentiallyexpressed.xlsx

- supplementalfigures.docx

- Supplementaltable2.ChIPQPCRprimers.xlsx 